\title{
Automorphic Systems and Lie-Vessiot Systems
}

\author{
By \\ Kazushige UENo
}

\section{Introduction}

In the theory of systems of partial differential equations, problems of integration of a given system have long occupied an important position.

Such are the problem of deciding whether integration of a given system could be reduced to those of several systems of ordinary differential equations or not and the problem of achieving integration of a given system, provided that the above reduction is possible.

S. Lie studied such a system of partial differential equations that a general solution of the system depends on a finite number of constant parameters. He reduced there the problem to the case of an involutive distribution. But his explanation of the method of the reduction is quite ambiguous (p. 115 in [2]). He carried out further reduction of integration of the involutive distribution to that of a 1-dimensional distribution according to Mayer's method.

Regarding these reductions as a fait accompli, he studied in [2] mainly integration of a 1-dimensioal distribution, which contains the study of integration of a non-linear ordinary differential equation of any order. From a standpoint of the theory of integration, he tried to classify ordinary differential equations in another paper. In [2] he studied the case that a 1-dimensional distribution has some connection with a continuous transformation group of finite type. The case that a group is simple and, in particular, isomorphic to the projective transformation group was investigated in detail by him. In the case that a group is solvable, integration of the distribution is deeply connected with quadrature, as is suggested by 
E. Cartan in $[1]$.

On the other hand, E. Vessiot studies the method of finding out integral curves of a given 1-dimensional distribution (which is, in a sense, equivalent to finding out first integrals of a given 1-dimensional distribution) in [4]. In other words, with respect to a certain kind of systems of ordinary differential equations of 1-st order, he attempted to develop the theory analogous to Galois theory of algebraic equations. Vessiot considered transformation groups which, roughly speaking, act on unknown functions of a given system of differential equations.

Now we shall go back to such a system of partial differential equations of any order that a general solution depends on a finite number of constant parameters.

Considering a continuous group of finite type acting on unknown functions of a given system and strengthening the condition that a general solution depends on a finite number of constant parameters, we put the assumption that, by the action of the group on a special solution, we can obtain a general solution of a given system of partial differential equations, which Vessiot called an autmorphic system with respect to the group.

Our main purpose is first to make clear the obscure point in the treatment in [2] of reducing integration of a given autmorphic system to that of an involutive distribution and secondly to give, in a form of a necessary and sufficient condition, an interpretation of the solvability of $G$ by properties of integration of an automorphic system with respect to $G$.

In $\S 1$ we give the definition of a Lie-Vessiot system $D$ on a principal fiber bundle $P(M, G, \pi)$ (Definition 1.1) and then we prove, in a strict form, the theorem stated in [1], which means that, if a Lie-Vessiot system is solvable, then it is integrated by quadratures (Theorem 1.1).

In $\S 2$ we define at first a $G$-autmorphic system (Definition 2.3). Under some general conditions we can induce a $G$-automorphic system with desirable properties from the given $G$-automorphic system such that the former is equivalent to the latter (Proposition 2.1, Proposition 2.4). We define the solvability of such a $G$-autmorphic system from a standpoint 
of integration (Definition 2.8). For such a $G$-automorphic system $(A)_{l}$ on $J^{l}(N, Q)$, we give a reduction theorem which reduce the integration of $(A)_{l}$ to that of a Lie-Vessiot system $D$ on $P(N, G, \pi)$ (Theorem 2.1). Using this theorem, we obtain the main theorem (Theorem 2.2) which gives an interpretation of the solvability of $G$ by that of a $G$-autmorphic system.

As for integration problems, not the existence or the property of solutions but the method of obtaining a solution is a question, though of course we need to certify the existence of a solution. S. Lie fixed his eyes upon a continuous transformation group as one of languages which express the necessary method to obtain a solution. It is also the origin of the notion of a Lie group. This language is very much available in some cases and express the properties of integration briefly. But of course this language is not all mighty.

We have written this note, taking his great thought as a starting point of our studies.

Finally, we should like to thank Professor N. Tanaka for his many valuable suggestions by reading our manuscript carefully.

\section{$\S 1$. Lie-Vessiot Systems}

We assume that the differentiability is the class $C^{\infty}$ and "a Lie group" always means "a connected Lie group" through this paper unless otherwise stated.

We denote by $P(M, G, \pi)$ a principal fiber bundle over the base space $M$, with the total space $P$, the structure group $G$ and the projection $\pi$.

Let $P(M, G, \pi)$ be a principal fiber bundle and let $g$ be the Lie algebra of $G$. Then for each $X \in \mathrm{g}, \exp t X$ induces a vector field $X^{*}$ on $P$. We set $\mathrm{g}^{*}=\left\{X^{*} \mid X \in \mathrm{g}\right\}$. Clearly $\mathrm{g}^{*}$ is a Lie algebra isomorphic to $\mathrm{g}$.

Definition 1.1. Let $P(M, G, \pi)$ be a principal fiber bundle. A distribution $D$ defined on a neighbourhood of $p \in P$ is called a Lie-Vessiot system at $p$ on $P(M, G, \pi)$ if it satisfies the following conditions;

(1) $D$ is an $m$-dimensional involutive distribution, $m=\operatorname{dim} M$. 
(2) $\pi_{*} D_{p}=T_{\pi(p)}(M)$

(3) $[X, Y]$ is a cross-section of $D$ for any cross-section $X$ of $D$ and any $Y \in \mathrm{g}^{*}$.

Note that, for any Lie-Vessiot system $D$ at $p$ on $P(M, G, \pi)$, there exists a local basis $\left\{X_{i}, \ldots, X_{m}\right\}$ of $D$ at $p$ such that $\left[X_{i}, X_{j}\right]=0(i, j=$ $1 \ldots m)$ and $\left[X_{j}, g^{*}\right]=0(j=1, \ldots, m)$.

Definition 1.2. A Lie-Vessiot system $D$ at $p$ on $P(M, G, \pi)$ is said to be simple (resp. solvable) if $G$ is simple (resp. solvable).

Defimition 1.3 . Let $D$ be any distribution on a manifold $S$. A function $\varphi$ locally defined at $p \in S$ is called a first integral of $D$ at $p$ if, for any local cross-section $X$ of $D$ at $p$, we have $X \cdot \varphi=0$.

Definition H.4. Let $_{\text {. }} D$ be a distribution on $S$. By the integration of $D$ at $p \in S$ we mean to find all first integrals of $D$ at $p$.

Definition 1.5. Let $D$ be an $m$-dimensional involutive distribution on $S$. A family $\left\{\varphi^{j}\right\}_{j=1}^{r}$ of first integrals of $D$ at $p$ is called a fundamental system of solutions of $D$ at $p$ if $d \varphi^{1}, \ldots, d \varphi^{r}$ are linearly independent at $p$ and $r=\operatorname{dim} S-m$.

Let $\left\{a_{h}\right\}_{h=1}^{k-1}$ be a family of real numbers. If we are given a family of real-valued functions $\left\{f_{h}\right\}_{h=1}^{k}$ defined on an open set $U$ of a manifold, we set $f=\left(f_{1}, \cdots, f_{k}\right), U_{f}^{l}=\left\{q \in U_{f}^{l-1} \mid f_{l}(q)=a_{l}\right\}$ and $f_{j}^{l}=f_{j}^{l-1} \mid U_{f}^{l}(l=$ $0, \ldots, k-1)$ where $U_{f}^{0}=U$ and $f_{j}^{0}=f_{j}$.

Theorem 1.1 . Let $D$ be a solvable Lie-Vessiot system at $p$ on $P(M$, $G, \pi), \operatorname{dim} G=r . \quad$ Then there exist a fundamental system of solutions $\left\{\varphi_{k}\right\}_{k=1}^{r}$ of $D$ at $p$ defined on $U$ and a basis $\left\{V_{k}\right\}_{k=1}^{r}$ of $g^{*}$ such that we have

$$
\begin{aligned}
& V_{l+1} \cdot \varphi_{l+1}=1 \\
& V_{l+k} \cdot \varphi_{l+1}=0 \quad(2 \leqq k \leqq r-l)
\end{aligned}
$$

for $0 \leqq l \leqq r-1$. 
Proof. Since $\mathrm{g}^{*}$ is solvable, we have a sequence of subalgebras $\mathrm{g}^{*}=$ $\mathrm{g}_{0}^{*} \supset \mathrm{g}_{1}^{*} \supset \cdots \mathrm{g}_{r-1}^{*} \supset \mathrm{g}_{r}^{*}=\{0\}$ where $\operatorname{dim} \mathrm{g}_{j}^{*}-\operatorname{dim} \mathrm{g}_{j+1}^{*}=1$ and $\mathrm{g}_{j+1}^{*}$ is an ideal of $\mathrm{g}_{j}^{*}$. Let $\left\{V_{k}\right\}_{k=1}^{r}$ be a basis of $\mathrm{g}^{*}$ such that $\left\{V_{k}\right\}_{k=j}^{r}$ is a basis of $\mathrm{g}_{j-1}^{*}$. Let $\left\{\phi_{k}\right\}_{k=1}^{r}$ be a fundamental system of solutions of $D$ at $p$ defined on $U$ such that $X_{h^{\circ}} \psi_{j}=V_{j+k^{\circ}} \psi_{j}=0(1 \leqq j \leqq r, 1 \leqq k \leqq r-j)$ where $\left\{X_{h}\right\}_{h=1}^{m}$ is any local basis of $D$ at $p$. The existence of such $\left\{\psi_{k}\right\}_{k=1}^{r}$ is assured, for $X_{1}, \ldots, X_{m}, V_{j+1}, \ldots, V_{r}$ generate an $(m+r-j)$-dimensional involutive distribution defined on a neighbourhood $U$ of $p$. Using the existence of such $\left\{\psi_{k}\right\}_{k=1}^{r}$, we shall show that there exists a fundamental system of solutions $\left\{\varphi_{k}\right\}_{k=1}^{r}$ of $D$ at $p$ defined on $U$ such that we have

$$
\left\{\begin{array}{l}
X_{l \cdot} \cdot \varphi_{l+1}=V_{l+k^{\circ}} \varphi_{l+1}=0 \quad(1 \leqq h \leqq m, 2 \leqq k \leqq r-l) \\
V_{l+1} \cdot \varphi_{l+1}=1
\end{array}\right.
$$

for $0 \leqq l \leqq r-1$. Since $\phi_{1}$ satisfies $X_{h} \cdot \psi_{1}=V_{k^{\circ}} \psi_{1}=0 \quad(1 \leqq h \leqq m, 2 \leqq k$ $\leqq r)$ and $\mathrm{g}_{1}^{*}$ is an ideal of $\mathrm{g}^{*}, V_{1}{ }^{\circ} \psi_{1}$ also satisfies $X_{h}\left(V_{1} \varphi_{1}\right)=V_{k}\left(V_{1} \bullet \psi_{1}\right)$ $=0(1 \leqq h \leqq m, 2 \leqq k \leqq r)$. Therefore we have a function $K(t)$ of one variable such that $V_{1} \bullet \psi_{1}=K\left(\phi_{1}\right)$. We set $H(t)=\int_{0}^{t} K(t)^{-1} d t$. Then $H\left(\phi_{1}\right)$ also satisfies $X_{h}\left(H\left(\psi_{1}\right)\right)=V_{k}\left(H\left(\phi_{1}\right)\right)=0(1 \leqq h \leqq m, 2 \leqq k \leqq r)$. Moreover we have $V_{1}\left(H\left(\psi_{1}\right)\right)=\left(\frac{d H}{d t}\right)_{t=\phi_{1}} \cdot V_{1}\left(\phi_{1}\right)=1$. Therefore we see that the system of partial differential equations

$$
\left\{\begin{array}{l}
X_{h} \cdot f=V_{k} \cdot f=0 \quad(1 \leqq h \leqq m, 2 \leqq k \leqq r) \\
V_{1} \circ f=1
\end{array}\right.
$$

has a solution on $U$. We may assume that $\pi^{-1}(J)=U \approx J \times W$ and let $\left\{x_{h}\right\}_{h}^{m}\left(\right.$ resp. $\left.\left\{w_{k}\right\}_{k=1}^{r}\right)$ be a coordinate system on $J$ (resp. $W$ ) Then $\left\{x_{1}\right.$, $\left.\ldots, x_{m}, w_{1}, \ldots, w_{r}\right\}$ is a coordinate system on $U$. By using this coordinate system, the above $(*)_{1}^{0}$ is expressed as

$(*)_{2}^{0}$

$$
\begin{cases}\sum \alpha_{h}^{i} \frac{\partial f}{\partial x_{1}}+\sum \beta_{h}^{j} \frac{\partial f}{\partial w_{j}}=0 & (1 \leqq h \leqq m) \\ \sum \gamma_{k}^{j} \frac{\partial f}{\partial w_{j}}=0 & (2 \leqq k \leqq r) \\ \sum \gamma_{1}^{j} \frac{\partial f}{\partial w_{j}}=1 . & \end{cases}
$$


Since $\left\{X_{1}, \ldots, X_{m}, V_{1}, \ldots, V_{r}\right\}$ are linearly independent on $U$, we can solve $(*)_{2}^{0}$ with respect to $\frac{\partial f}{\partial x_{h}}, \frac{\partial f}{\partial w_{k}}(1 \leqq h \leqq m, 1 \leqq k \leqq r)$. We have

$(*)_{3}^{0} \quad \begin{cases}\frac{\partial f}{\partial x_{h}}=\rho_{h}^{0}\left(x_{1}, \ldots, x_{m}, w_{1}, \ldots, w_{r}\right) & (1 \leqq h \leqq m) \\ \frac{\partial f}{\partial w_{k}}=\sigma_{k}^{0}\left(x_{1}, \ldots, x_{m}, w_{1}, \ldots, w_{r}\right) & (1 \leqq k \leqq r) .\end{cases}$

Now we assume that we have linearly independent functions $\varphi_{1}, \cdots, \varphi_{l}$ on $U$ such that

$$
\left\{\begin{array}{l}
X_{h} \cdot \varphi_{j}=V_{j+k} \cdot \varphi_{j}=0 \quad(1 \leqq h \leqq m, 1 \leqq k \leqq r-j) \\
V_{j} \cdot \varphi_{j}=1
\end{array}\right.
$$

for $1 \leqq j \leqq l$. Then we shall show that we can find a function $\varphi_{l+1}$ on $U$ which is independent of $\varphi_{1}, \cdots, \varphi_{l}$ and satisfies

$$
\left\{\begin{array}{l}
X_{l} \cdot \varphi_{l+1}=V_{l+k} \cdot \varphi_{l+1}=0 \quad(1 \leqq h \leqq m, 2 \leqq k \leqq r-l) \\
V_{l+1} \cdot \varphi_{l+1}=1
\end{array}\right.
$$

Since $\varphi_{j}(1 \leqq j \leqq l)$ satisfies $X_{h^{\circ}} \varphi_{j}=V_{j+k} \varphi_{j}=0 \quad(1 \leqq h \leqq m, 1 \leqq k \leqq r-j)$, we can restrict $X_{h}(1 \leqq h \leqq m)$ (resp. $V_{l+k}(1 \leqq k \leqq r-l)$ ) to $U_{\varphi}^{l}$ which we denote by $X_{h}^{l}$ (resp. $V_{l+k}^{l}$ ). By the same reason as for the case $(*)_{1}^{0}$, we can induce a function $\varphi_{l+1}^{l}$ on $U_{\varphi}^{l}$ from the function $\psi_{l+1}^{l}\left(=\psi_{l+1} \mid U_{\varphi}^{l}\right)$ such that we have $X_{h}^{l} \cdot \varphi_{l+1}^{l}=V_{l+k}^{l} \cdot \varphi_{l+1}^{l}=0 \quad(1 \leqq h \leqq m, 2 \leqq k \leqq r-l)$ and $V_{l+1}^{l} \varphi_{l+1}^{l}=1$, that is to say, we can see that the system of partial differential equations

$(*)_{1}^{l}$

$$
\left\{\begin{array}{l}
\mathrm{X}_{h}^{l} \cdot f=V_{l+k}^{l} \cdot f=0 \quad(1 \leqq h \leqq m, 2 \leqq k \leqq r-l) \\
V_{l+1}^{l} \cdot f=1
\end{array}\right.
$$

has a solution on $U_{\varphi}^{l}$.

Now we may consider $\left\{x_{1}, \ldots, x_{m}, w_{1}, \ldots, w_{r-l}, \varphi_{1}, \ldots, \varphi_{l}\right\}$ as a coordinate system on $U$. Then $\left\{x_{1}, \ldots, x_{m}, w_{1}, \ldots, w_{r-l}\right\}$ is a coordinate system on $U_{\varphi}^{l}$. By using the coordinate system on $U_{\varphi}^{l}$, we have 
$(*)_{2}^{\frac{1}{2}}$

$$
\left\{\begin{array}{l}
\sum_{i=1}^{m} \alpha_{h}^{i, l} \frac{\partial f}{\partial x_{i}}+\sum_{j=1}^{r} \beta_{h}^{j, l} \frac{\partial f}{\partial w_{j}}=0 \quad(1 \leqq h \leqq m) \\
\sum_{j=1}^{r} \gamma_{k}^{j, l} \frac{\partial f}{\partial w_{j}}=0 \quad(l+2 \leqq k \leqq r) \\
\sum_{j=1}^{r} \gamma_{l+1}^{j, l} \frac{\partial f}{\partial w_{j}}=1 .
\end{array}\right.
$$

Since $\mathrm{X}_{h}^{l}, V_{l+k}^{l}(1 \leqq h \leqq m, 1 \leqq k \leqq r-l)$ are linearly independent on $U^{l}$, we can solve $(*)_{2}^{l}$ with respect to $\frac{\partial f}{\partial x_{h}}, \frac{\partial f}{\partial w_{k}}(1 \leqq h \leqq m, 1 \leqq k \leqq r-l)$. We have then,

$(*)_{3}^{l}$

$$
\begin{cases}\frac{\partial f}{\partial x_{h}}=\rho_{h}^{l}\left(x_{1}, \cdots, x_{m}, w_{1}, \cdots, w_{r-l}, \varphi_{1}, \cdots, \varphi_{l}\right) & (1 \leqq h \leqq m) \\ \frac{\partial f}{\partial w_{k}}=\sigma_{k}^{l}\left(x_{1}, \cdots, x_{m}, w_{1}, \cdots, w_{r-l}, \varphi_{1}, \cdots, \varphi_{l}\right) & (1 \leqq k \leqq r-l),\end{cases}
$$

$\rho_{h}^{l}$ and $\sigma_{k}^{l}$ are differentiable with respect to $x_{1}, \ldots, x_{m}, w_{1}, \ldots, w_{r-l}, \varphi_{1}, \ldots$, $\varphi_{l}$. For any family of real numbers $\left\{a_{j}\right\}_{j=1}^{l},(*)_{3}^{l}$ has a solution on $U_{\varphi}^{l}$. Therefore $(*)_{3}^{l}$ has a solution $\varphi_{l+1}$ on $U$. Clearly $\varphi_{l+1}$ satisfies

$$
\left\{\begin{array}{l}
\mathrm{X}_{h^{\circ}} \varphi_{l+1}=V_{l+k} \cdot \varphi_{l+1}=0 \quad(1 \leqq h \leqq m, 2 \leqq k \leqq r-l) \\
V_{l+1} \cdot \varphi_{l+1}=1 .
\end{array}\right.
$$

Thus we get a fundamental system of solutions $\left\{\varphi_{j}\right\}_{j=1}^{r}$ of $D$ at $p$ such that we have

$$
\left\{\begin{array}{l}
V_{l+1^{\circ}} \varphi_{l+1}=1 \\
V_{l+k^{\circ}} \varphi_{l+1}=0 \quad(2 \leqq k \leqq r-l) .
\end{array}\right.
$$

This completes the proof of Theorem 1.1.

Corollary 1.1. Let $D$ be a solvable Lie-Vessiot system at $p$ on $P(M$, $G, \pi)$. Then we can find a fundamental system of solutions $\left\{\varphi_{k}\right\}_{k=1}^{r}$ of $D$ at $p$ by quadratures.

Proof. By the proof of Theorem 1.1, there exists a fundamental system of solutions $\left\{\varphi_{k}\right\}_{k=1}^{r}$ of $D$ at $p$ such that we have 
$(*)_{3}^{l}$

$$
\begin{cases}\frac{\partial \varphi_{l+1}}{\partial x_{h}}=\rho_{h}^{l}\left(x_{1}, \ldots, x_{m}, w_{1}, \ldots, w_{r-l}, \varphi_{1}, \ldots, \varphi_{l}\right) & (1 \leqq h \leqq m) \\ \frac{\partial \varphi_{l+1}}{\partial w_{k}}=\sigma_{k}^{l}\left(x_{1}, \cdots, x_{m}, w_{1}, \cdots, w_{r-l}, \varphi_{1}, \cdots, \varphi_{l}\right) & (1 \leqq k \leqq r-l)\end{cases}
$$

for $0 \leqq l \leqq r-1$, which we can integrate by quadratures according to the method due to Lagrange and Charpit.

\section{§2. Solvability of Automorphic Systems of Finite Type}

Let $N$ and $Q$ be manifolds. We denote by $J^{l}(N, Q)$ the space of $l$-jets of local maps of $N$ to $Q$. Let $s$ be any map of a neighbourhood $U_{x_{0}}$ of $x_{0} \in N$ to $Q$ and set $j_{x}^{l}(s)=\left(x, s(x), s_{(1)}(x), \ldots, s_{(l)}(x)\right)$ where $s_{(k)}$ is the set of partial derivatives of $s$ of order $k$. If $p$ is in $J^{l}(N, Q)$, we have $p=j_{x}^{l}(s) . \quad x$ (resp. $s(x)$ ) is called the source of $p$ (resp. the target of $p$ ). For a map $\bar{s}$ of $U_{x_{0}} \times V_{e} \subset N \times G$ to $Q$ where $V_{e}$ is a neighbourhood of the unit element $e$ of a Lie group $G$, we set $s_{g}(x)=\bar{s}(x, g)$ and define $j^{l}(\bar{s})$ by $j^{l}(\bar{s})(x, g)=j_{x}^{l}\left(s_{g}\right)$. Then $j^{l}(\bar{s})$ is a map of $U_{x_{0}} \times V_{e}$ to $J^{l}(N, Q)$.

In this section we assume that $N=\mathbb{R}^{n}$ and $Q=\mathbb{R}^{q}$. We denote by $x_{1}, \ldots, x_{n}$ the coordinate system of $N$, by $z_{1}, \ldots, z_{q}$ that of $Q$ and by $x_{i}(1 \leqq i \leqq n), z_{j}(1 \leqq j \leqq q), p_{j_{1} \ldots j_{k}}^{\lambda}\left(1 \leqq \lambda \leqq q, 1 \leqq j_{1}, \cdots, j_{k} \leqq n, 1 \leqq k \leqq l\right)$ that of $J^{l}(N, Q)$.

Definition 2.1. Let $\left\{F_{j}\right\}_{j=1}^{\alpha}$ be a family of functions defined on a neighbourhood of $p_{0} \in J^{k}(N, Q)$. A system of equations

$$
(A)_{k}: \quad F_{1}=0, \ldots, F_{\alpha}=0
$$

is called a system of partial differential equations at $p_{0} \in J^{k}(N, Q)$.

We denote by $I(A)_{k}$ the set of points in $J^{k}(N, Q)$ satisfying $(A)_{k}$.

Definition 2.2. Let $x_{0}$ be the source of $p_{0}$. Any local map $s$ of a neighbourhood $U_{x_{0}}$ of $x_{0}$ to $Q$ is called a solution of $(A)_{k}$ if $j^{l}(s)\left(U_{x_{0}}\right)=$ $\left\{j_{x}^{l}(s) \mid x \in U_{x_{0}}\right\}$ is contained in $I(A)_{k}$.

Let $(A)_{k}: \quad F_{1}=0, \ldots, F_{\alpha}=0$ be a system of partial differential equa- 
tions given on a neighbourhood $U$ of $p_{0} \in J^{k}(N, Q)$. We denote by $Q^{k}(U)$ the sheaf of all local functions on $U$ and by $(A)_{k}^{*}$ the sheaf of ideals of $Q^{k}(U)$ generated by $F_{1}, \ldots, F_{\alpha}$. Moreover let $\rho_{k}^{k+1}$ be the projection of $J^{k+1}(N, Q)$ onto $J^{k}(N, Q)$ and we denote by $p(A)_{k}^{*}$ the sheaf of ideals of $Q^{k+1}(\widetilde{U}), \tilde{U}=\left(\rho_{k}^{k+1}\right)^{-1}(U)$, generated by $(A)_{k}^{*}$ and $\partial_{\sharp i}^{i} F_{j}(1 \leqq i \leqq n, 1 \leqq j \leqq$ $\alpha$ ) where $\partial_{\sharp}^{i} F_{j}$ is defined by

$$
\partial \partial_{\sharp} F_{j}=\frac{\partial F_{j}}{\partial x_{i}}+\sum_{\lambda} \frac{\partial F_{j}}{\partial z_{\lambda}} p_{i}^{\lambda}+\cdots+\sum_{\lambda, j_{1} \ldots, j_{k}} \frac{\partial F_{j}}{\partial p_{j_{1} \ldots j_{k}}^{\lambda}} p_{j_{1} \ldots j_{k} i}^{\lambda}
$$

$p(A)_{k}^{*}$ is called the prolongation of $(A)_{k}^{*}$. We set $p_{0}(A)_{k}^{*}=Q^{l}(U) \cap p(A)_{k}^{*}$ and $\overline{(A)_{k}^{*}}=\bigcup_{n=1}^{\infty} p_{0}^{n}(A)_{k}^{*}, p_{0}^{n}(A)_{k}^{*}=p_{0}\left(p_{0}^{n-1}(A)_{k}^{*}\right)$. M. Matsuda called $\overline{(A)_{k}^{*}}$ the $p$-closure of $(A)_{k}^{*}$.

Now let $G$ be a Lie transformation group acting effectively on $Q$. For any $p=j_{x}^{k}(s) \in J^{k}(N, Q)$ and $g \in G$, we set $g \circ j_{x}^{k}(s)=j_{x}^{k}(g \circ s)$. Then $G$ acts on $J^{k}(N, Q)$ as an effective Lie transformation group.

Definition 2.3. Let $G$ be a Lie transformation group acting effectively on $Q$. A system of partial differential equations $(A)_{k}$ at $p_{0} \in$ $J^{k}(N, Q)$ is said to be $G$-autmorphic if there exists a map $\bar{s}$ of $U_{x_{0}} \times$ $V_{e} \subset N \times G$ to $Q\left(x_{0}=\right.$ the source of $\left.p_{0}\right)$ satisfying the following conditions;

(1) For any $g \in V_{e}, s_{g}$ is a solution of $(A)_{k}$.

(2) Any solution of $(A)_{k}$ is uniquely expressed as $s_{g}, g \in V_{e}$.

(3) We have $\bar{s}(x, g)=g \cdot \bar{s}(x, e)$ for any $g \in V_{e}$.

We call such a map $\bar{s}$ a general solution of the $G$-autmorphic system $(A)_{k}$.

Remark 2.1. We have $g \cdot j^{k}(\bar{s})(x, e)=j^{k}(\bar{s})(x, g)$ for any $g \in V_{e}$.

We denote by $\left(\begin{array}{l}\lambda \\ j_{1} \ldots j_{s}\end{array}\right)$ the pair of an integer $\lambda$ and a family of integers $\left\{j_{h}\right\}_{h=1}^{s}$. We set $\tilde{I}^{(l)}=\left\{\left(\left(_{j_{1} \ldots j_{s}}^{\lambda}\right) \mid 1 \leqq \lambda \leqq q, 1 \leqq s \leqq l, 1 \leqq j_{h} \leqq n\right\}\right.$ where $q=\operatorname{dim} Q, n=\operatorname{dim} N$.

Definition 2.4. A system of partial differential equations $(A)_{k}$ at $p$ in $J^{k}(N, Q)$ is said to be of normal form if $(A)_{k}$ possesses the form $p_{j_{1} \ldots j_{s}}^{\lambda}$ $=H_{j_{1} \ldots j_{s}}^{\lambda}\left(x_{1}, \ldots, x_{n}, z_{1}, \ldots, z_{q}, p_{1}^{1}, \ldots\right),\left(\lambda_{j_{1} \ldots j_{s}}^{\lambda}\right) \in I$ where $I$ is a subset of 
$\tilde{I}^{(k)}$ satisfying the following conditions;

(1) We set $I_{k}=\left\{\left({ }_{j_{1} \ldots j_{k}}^{\lambda}\right) \mid 1 \leqq \lambda \leqq q, 1 \leqq j_{h} \leqq n\right\}$.

Then we have $I \supset I_{k}$.

(2) $H_{j_{1} \ldots j_{s}}^{\lambda}$ is a function on $J^{k-1}(N, Q)$ for any $\left(\begin{array}{l}\lambda \\ j_{1} \ldots j_{s}\end{array}\right) \in I$.

(3) For any $\left(\begin{array}{l}\lambda \\ j_{1} \ldots j_{s}\end{array}\right) \in I, H_{j_{1} \ldots j_{s}}^{\lambda}$ does not depend on $p_{j_{1} \ldots j_{s}}^{\lambda}$.

In order to emphasize that $(A)_{k}$ is of normal form, we denote by $\mathfrak{N}(A)_{k}$ in place of $(A)_{k}$.

Proposition 2.1. Let $(A)_{k}$ be a system of partial differential equations at $p_{0} \in J^{k}(N, Q)$ satisfying the following conditions;

(1) $(A)_{k}$ is G-automorphic.

(2) There exists a general solution $\bar{s}$ of $(A)_{k}$ such that, for an integer $l \geqq k, j^{l}(\bar{s})$ is an embedding of a neighbourhood $U_{x_{0}} \times V_{e}$ of $\left(x_{0}, e\right) \in$ $N \times G$ into $J^{l}(N, Q)$.

Then there exists a system of partial differential equations $(A)_{l+1}$ at $\tilde{p}_{0}$ in $J^{l+1}(N, Q)$ with $\rho_{k}^{l+1}\left(\tilde{p}_{0}\right)=p_{0}\left(\rho_{k}^{l+1}\right.$ is the projection of $J^{l+1}(N, Q)$ onto $\left.J^{k}(N, Q)\right)$ satisfying the following conditions;

(i) $(A)_{l+1}$ is G-automorphic and has a general solution $\bar{\omega}: U_{x_{0}} \times$ $V_{e} \supset U_{x_{0}}^{\prime} \times V_{e}^{\prime} \rightarrow Q$ with $\bar{\omega}=\bar{s} \mid U_{x_{0}}^{\prime} \times V_{e}^{\prime}$.

(ii) There exists a neighbourhood $W$ of $\tilde{p}_{0}$ such that $I\left((A)_{l+1}\right) \cap$ $W=\tilde{S} \cap W$ where $\tilde{S}=j^{l+1}(\bar{s})\left(U_{x_{0}} \times V_{e}\right)$.

(iii) $(A)_{l+1}$ contains, as a subsystem, a system of partial differential equations $\mathfrak{N}(B)_{l}$ of normal form.

Proof. We may consider that $\left\{x_{1}, \ldots, x_{n}, z_{1}, \ldots, z_{t}, w_{1}, \ldots, w_{r-t}\right\}$ is a local coordinate system at $\bar{p}_{0}$ in $S=j^{l}(s)\left(U_{x_{0}} \times V_{e}\right), \rho_{l}^{l+1}\left(\tilde{p}_{0}\right)=\bar{p}_{0}$, where $r=\operatorname{dim} G$ and $w_{i}(1 \leqq j \leqq r-t)$ is some $p_{j_{1} \ldots j_{s}}^{\lambda}$. Since $\widetilde{S}$ is diffeomorphic to $S$ by the projection $\rho_{l}^{l+1}$ of $J^{l+1}(N, Q)$ onto $J^{l}(N, Q)$, we may also consider $\left\{x_{1}, \ldots, x_{n}, z_{1}, \ldots, z_{t}, w_{1}, \ldots, w_{r-t}\right\}$ as a local coordinate system at $\tilde{p}_{0}$ in $\widetilde{S}$. Let $p_{j_{1} \ldots j_{s}}^{\lambda}(1 \leqq s \leqq l+1)$ be any coordinate function on $J^{l+1}(N, Q)$ such that $p_{j_{1} \ldots j_{s}}^{\lambda} \neq w_{j}(j=1, \ldots, r-t)$. Then we have $p_{j_{1} \ldots j_{s}}^{\lambda}=$ $H_{j_{1} \ldots j_{s}}^{\lambda}\left(x_{1}, \ldots, x_{n}, z_{1}, \ldots, z_{t}, w_{1}, \ldots, w_{r-t}\right)$ on a neighbourhood $U_{\tilde{p}_{0}}$ of $\tilde{p}_{0}$ in $\tilde{S}$. Similarly if $z_{i} \neq z_{h}(h=1, \ldots, t)$, then we have $z_{i}=H^{i}\left(x_{1}, \ldots, x_{n}, z_{1}\right.$, 
$\left.\ldots, z_{t}, w_{1}, \ldots, w_{r-t}\right)$. We denote by $(A)_{l+1}$ the system of partial differential equations consisting of all such $p_{j_{1} \ldots j_{s}}^{\lambda}=H_{j_{1} \ldots j_{s}}^{\lambda}$ and $z_{i}=H^{i}$. We denote also by $\mathfrak{R}(B)_{l+1}$ the system of partial differential equations consisting of all such $p_{j_{1} \ldots j_{s}}^{\lambda}=H_{j_{1} \ldots j_{s^{*}}}^{\lambda}$ Then $(A)_{l+1}$ clearly satisfies (ii) and (iii). We shall prove that $(A)_{l+1}$ satisfies (i).

First of all we shall show that if $s: U_{x_{0}}^{\prime} \rightarrow Q$ is a solution of $(A)_{l+1}$ then $s$ is a solution of $(A)_{k}$. Clearly $j^{l}(s)$ is a local cross-section of $S$. For each $x \in U_{x_{0}}^{\prime}, j_{x}^{l}(s) \in S$. Therefore we have a solution $s_{g(x)}, g(x) \in V_{e}$, of $(A)_{k}$ such that $j_{x}^{l}(s)=j_{x}^{l}\left(s_{g(x)}\right)$. In particular we have $j_{x}^{l}(s)=j_{x}^{k}\left(s_{g(x)}\right)$ for $k \leqq l$. Therefore $j_{x}^{k}(s) \in I(A)_{k}$. Since $x$ is any point in $U_{x_{0}}^{\prime}, s$ is a solution of $(A)_{k}$. Next assume that $s$ is a solution of $(A)_{k}$. Then $j^{l}(s)$ is a local crosssection of $S$ and therefore $j^{l+1}(s)$ is a local cross-section of $\widetilde{S}$. This implies that $s$ is a solution of $(A)_{l+1}$.

Therefore $(A)_{l+1}$ has a general solution $\tilde{s} \mid U_{x_{0}}^{\prime} \times V_{e}^{\prime}$. It is now clear that $(A)_{l+1}$ is $G$-automorphic. This completes the proof of Proposition 2.1.

Proposition 2.2. Let $\mathfrak{N}(A)_{l}$ be a G-automorphic system at $p_{0} \in$ $J^{l}(N, Q)$. We denote by $\overline{\mathfrak{N}}(A)_{l}^{*}$ the p-closure of $\mathfrak{R}(A)_{l}^{*}$. We assume that the point $p_{0} \in \mathbb{I}\left(\overline{\mathfrak{N}}(A)_{l}^{*}\right)$ is an ordinary integral point of $\overline{\mathfrak{N}}(A)_{l}^{*}$. Then there exists a neighbourhood $U_{p_{0}}$ of $p_{0}$ in $J^{l}(N, Q)$ such that $\overline{\Re(}(A)_{l}^{*}$ is involutive at $p \in I\left(\overline{\mathfrak{N}}(A)_{l}^{*}\right) \cap U_{p_{0}}$. (As for the definition of "(quasi-) involutive" confer [3].)

Proof. Since, for a suitable $U_{p_{0}}, p \in I\left(\overline{\mathfrak{N}}(A)_{l}^{*}\right) \cap U_{p_{0}}$ is an ordinary integral point of $\overline{\mathfrak{R}}(A)_{l}^{*}$ and $\overline{\mathfrak{N}}(A)_{l}^{*}$ is compatible at $p \in I\left(\overline{\mathfrak{N}}(A)_{l}^{*}\right) \cap U_{p_{0}}$, we have only to show that $C_{p}\left(\overline{\mathfrak{N}}(A)_{l}^{*}\right)$ is involutive and the dimension of $C_{p}\left(\overline{\mathfrak{N}}(A)_{l}^{*}\right)^{(1)}$, the first prolongation of $C_{p}\left(\overline{\mathfrak{N}}(A)_{l}^{*}\right)$, is locally constant at p. By definition we have $C_{p}\left(\overline{\mathfrak{Y l}}(A)_{l}^{*}\right)=\left\{\mathrm{X} \in T_{p}\left(J^{l}(N, Q)\right) \mid\left(\rho_{l-1}^{l}\right)_{*} \mathrm{X}=0\right.$, $\left.d f_{p}(\mathrm{X})=0,{ }^{\forall} f \in \bar{\Re}(A)^{*}\right\}=\left\{\sum_{\lambda, j_{1} \ldots, j_{l}} \xi_{j_{1} \ldots j_{l}}^{\lambda}(p) \frac{\partial}{\partial p_{j_{1} \ldots j_{l}}^{\lambda}} \mid\left(\sum_{\lambda, j_{1}, \ldots, j_{l}} \xi_{j_{1} \ldots j_{l}}^{\lambda_{l}} \frac{\partial}{\partial p_{j_{1} \ldots j_{l}}^{\lambda}}\right)\right.$ $\left.{ }_{p} f=0,{ }^{\forall} f \in \overline{\mathfrak{N}}(A)_{l}^{*}\right\}$. On the other hand $\overline{\mathfrak{N}}(A)_{l}^{*}$ contains $p_{j_{1} \ldots j_{l}}-H_{j_{1} \ldots j_{l}}$ for any $\left({ }_{j_{1} \ldots j_{l}}^{\lambda}\right) \in I_{l}$. It follows immediately that $C_{p}\left(\bar{\Re}(A)_{l}^{*}\right)=0$. Therefore, in particular, $\operatorname{dim} C_{p}\left(\overline{\mathfrak{N}}(A)_{l}^{*}\right)^{(1)}=$ constant and $C_{p}\left(\overline{\mathfrak{Y}}(A)_{l}^{*}\right)$ is involutive. This completes the proof of Proposition 2.2. 
Let $\mathfrak{N}(A)_{l}$ be a system at $p_{0} \in J^{l}(N, Q)$. For any $H_{j_{1} \ldots j_{s+1}}^{\lambda}$ which appears in $\mathfrak{N}(A)_{l}$, we define a function ${ }_{i} H_{j_{1} \ldots j_{s+1}}^{\lambda}$ on $J^{l}(N, Q)$ given in a neighbourhood of $p_{0}$ by the following way: For $\partial \partial_{\#}^{i} H_{j_{1} \ldots j_{s+1}}^{\lambda}=\frac{\partial H_{j_{1} \ldots j_{s+1}}^{\lambda}}{\partial x_{i}}+$ $\sum_{\mu} p_{i}^{\mu} \frac{H_{j_{1} \ldots j_{s+1}}^{\lambda}}{\partial z_{\mu}}+\cdots+\sum_{\mu, h_{1}, \ldots, h_{l-1}} p_{h_{1} \ldots h_{l-1}}^{\mu} i \frac{\partial H_{j_{1} \ldots j_{s+1}}^{\lambda}}{\partial p_{h_{1} \ldots h_{l-1}}^{\mu}}$, replace the coefficients $p_{h_{1} \ldots h_{k} i}^{\mu}$ of $\partial_{\sharp}^{i} H_{j_{1} \ldots j_{s+1}}^{\lambda}(0 \leqq k \leqq l-1)$ which appear in the left hand side of $\mathfrak{N}(A)_{l}$ by the right hand side of it, which we denote by ${ }_{i} H_{j_{1} \ldots j_{s+1}}^{\lambda}$. If both $\left({ }_{\alpha j_{1} \ldots j_{s}}^{\lambda}\right)$ and $\left({ }_{\beta j_{1} \ldots j_{s}}^{\lambda}\right)$ are in $I$, we consider the function ${ }_{\alpha} H_{\beta j_{1} \ldots j_{s}}^{\lambda}-$ ${ }_{\beta} H_{\alpha_{j_{1} \ldots j_{s}}}^{\lambda}$ and if $\left({ }_{\beta j_{1} \ldots j_{s}}^{\lambda}\right) \in I$, and $\left(\begin{array}{l}\lambda \\ \alpha_{j_{1} \ldots j_{s}}\end{array}\right) \notin I$, we consider the function ${ }_{\alpha} H_{\beta j_{1} \ldots j_{s}}^{\lambda}-P_{j_{1} \ldots j_{s} \alpha \beta}^{\lambda}$. We denote by $\mathfrak{F} \mathfrak{R}(A)_{l}$ the sheaf of rings of all such functions on a neighbourhood of $p_{0}$.

Proposition 2.3. Let $\mathfrak{N}(A)_{\text {l }}$ be a system at $p_{0} \in J^{l}(N, Q)$. We have, then, $I\left(\mathfrak{F} \mathfrak{R}(A)_{l}\right) \supset I\left(\overline{\mathfrak{N}}(A)_{l}^{*}\right)$.

Proof. By definition, $\overline{\mathfrak{N}}(A)_{l}^{*}$ contains $\partial_{\#}^{\alpha} H_{\beta j_{1} \ldots j_{l-1}}^{\lambda}-\partial_{\#}^{\beta} H_{\alpha j_{1} \ldots j_{l-1}}$ for any $\left(\begin{array}{l}\lambda \\ \alpha \beta j_{1} \ldots j_{l-1}\end{array}\right)$ and $p_{j_{1} \ldots j_{s}}^{\lambda}-H_{j_{1} \ldots j_{s}}^{\lambda}$ for any $\left({ }_{j_{1} \ldots j_{s}}^{\lambda}\right) \in I$. Moreover if $\left({ }_{\beta j_{1} \ldots j_{s}}^{\lambda}\right)$ $\in I$ and $\left(\begin{array}{l}\lambda \\ \alpha_{j_{1} \ldots j_{s}}\end{array}\right) \notin I$, then $\partial_{\#}^{\alpha} H_{j_{1} \ldots j_{s}}-p_{j_{1} \ldots j_{s} \alpha \beta}^{\lambda}$ is contained in $\overline{\mathfrak{R}}(A)_{l}^{*}$. Therefore we have $\mathfrak{F} \mathfrak{N}(A)_{l} \subset \overline{\mathfrak{N}}(A)_{l}^{*}$, that is, we get $I\left(\mathfrak{\Im} \mathfrak{N}(A)_{l}\right) \supset I\left(\overline{\mathfrak{N}}(A)_{l}^{*}\right)$.

Proposition 2.4. Let $\Re(A)_{l}$ be a $G$-automorphic system at $p_{0} \in J^{l}(N, Q)$ such that $j^{l}(\bar{s})$ is an embedding of $U_{x_{0}} \times V_{e}$ into $J^{l}(N, Q)$. We assume that the differentiability is the class $C^{\omega}$ and the point $p_{0} \in I\left(\overline{\mathfrak{N}}(A)_{l}^{*}\right)$ is an ordinary integral point of $\bar{\Re}(A)_{l}^{*}$. Then there exists a neighbourhood $U_{p_{0}}$ of $p_{0}$ in $J^{l}(N, Q)$ such that we have $I\left(\bar{\Re}(A)_{l}^{*}\right) \cap U_{p_{0}}=S \cap U_{p_{0}}$ where $S=$ $j^{l}(\bar{s})\left(U_{x_{0}} \times V_{e}\right)$.

Proof. By Proposition 2.2, we can choose a neighbourhood $U_{p_{0}}$ of $p_{0}$ in $J^{l}(N, Q)$ such that $\overline{\mathfrak{N}}(A)_{l}^{*}$ is involutive at any $p \in I\left(\overline{\mathfrak{N}}(A)_{b}^{*}\right) \cap U_{p_{0}}$. Therefore we have a solution $s: U^{\prime} \rightarrow Q$ of $\overline{\mathfrak{N}}(A)_{l}^{*}$ such that $j^{l}(s)\left(U^{\prime}\right)$ contains $p$. This implies $I\left(\overline{\mathfrak{R}}(A)_{l}^{*}\right) \cap U_{p_{0}} \subset S \cap U_{p_{0}}$. On the other hand we have clearly $S \cap U_{p_{0}} \subset I\left(\bar{\Re}(A)_{l}^{*}\right) \cap U_{p_{0}}$. Therefore we get $I\left(\overline{\mathfrak{R}}(A)_{b}^{*}\right) \cap U_{p_{0}}=$ $S \cap U_{p_{0}}{ }^{\circ}$

Therefore from now on we shall deal with a system of partial differ- 
ential equations $(A)_{l}$ at $p_{0} \in J^{l}(N, Q)$ satisfying the following conditions; $\left[\alpha_{1}\right](A)_{l}$ is $G$-automorphic and $j^{l}(\bar{s})$ is an embedding of $U_{x_{0}} \times V_{e}$ into $J^{l}(N, Q)$ where $\bar{s}: U_{x_{0}} \times V_{e} \rightarrow Q$ is a general solution of $(A)_{l}$.

$\left[\alpha_{2}\right]$ There exists a neighbourhood $W$ of $p_{0}$ such that $S \cap W=I(A)_{l}$ $\cap W$ where $S=j^{l}(\bar{s})\left(U_{x_{0}} \times V_{e}\right)$.

$\left[\alpha_{3}\right](A)_{l}$ contains, as a subsystem, a system of partial differential equations $\mathfrak{N}(B)_{\text {l }}$ of normal form.

Moreover we set the following assumption;

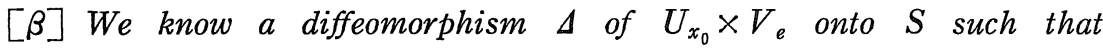
$g \cdot \Delta(x, e)=\Delta(x, g)$ for any $g \in V_{e}$ and $x \in U_{x_{0}}$.

For such a system of partial differential equations, we have the following reduction theorem.

Theorem 2.1. Let $(A)_{l}$ be a system of partial differential equations at $p_{0}$ satisfying $\left[\alpha_{i}\right](i=1,2,3)$ and $[\beta]$. Then we can induce from $(A)_{l}$ a Lie-Vessiot system $D$ at $q_{0}=\Delta^{-1}\left(p_{0}\right)$ on the trivial principal fiber bundle $(N \times G)(N, G, \pi)$ such that, for any first integral $\varphi$ of $D$ at $q_{0}, \varphi \circ \Delta^{-1}$ is constant on $S_{\omega}=\left\{j_{x}^{l}(\omega) \mid x \in U_{x_{0}}^{\prime}\right\}$ for any solution $\omega: U_{x_{0}}^{\prime} \rightarrow Q$ of $(A)_{l}$.

Proof. We set $E_{i}=\frac{\partial}{\partial x_{i}}+\sum_{\lambda} p_{i}^{\lambda} \frac{\partial}{\partial z_{\lambda}}+\cdots+\sum_{\lambda, j_{1}, \ldots, j_{l-1}} p_{j_{1} \ldots j_{l-1} i}^{\lambda} \frac{\partial}{\partial p_{j_{1} \ldots j_{l-1}}^{\lambda}}$. Then $E_{i}$ is a vector field on $J^{l}(N, Q)$. We replace the coefficients $p_{j_{1} \ldots j_{k} i}^{\lambda}$ of $E_{i}$ which appear in the left hand side of $\mathfrak{N}(B)_{l}$ by the right hand side of it. Then we obtain a new vector field $A_{i}$ on $J^{l-1}(N, Q)$ which can be regarded naturally as a vector field on $J^{l}(N, Q)$, for we have the assumption $N=\boldsymbol{R}^{n}$ and $Q=\mathbb{R}^{q}$. Moreover $A_{1}, \ldots, A_{n}$ are linearly independent at any point in $J^{l}(N, Q)$. Therefore they generate an $n(=\operatorname{dim} N)$ dimensional distribution $\widetilde{D}$ on $J(N, Q)$. Let $s: U_{x_{0}} \times V_{e} \rightarrow Q$ be a general solution of $(A)_{l}$ and set $S_{g}=\left\{j_{x}^{l}\left(s_{g}\right) \mid x U_{x_{0}}\right\}, g \in V_{e}$. By the construction of $A_{i}$, for a map $\omega: U_{x_{0}}^{\prime} \rightarrow Q, A_{i}$ is tangent to $S_{\omega}=\left\{j_{x}^{l}(\omega) \mid x U_{x_{0}}^{\prime}\right\}$ if $\omega$ is a solution of $(A)_{l}$. Therefore $A_{i}$ is tangent to $S_{g}$. Since we have $S=$ $\bigcup_{g \in V_{e}} S_{g}, \tilde{D}$ is tangent to $S$ at any point. By calculating $\left[A_{i}, A_{j}\right]$, it follows that $\left[A_{i}, A_{j}\right]_{p}=0$ if and only if $p \in I\left(\mathfrak{F} \mathfrak{N}(B)_{l}\right)$. Therefore by Pro- 
position 2.3 and $I\left(\overline{\mathfrak{N}}(B)_{l}^{*}\right) \supset S$ the restriction $D^{S}$ of $\tilde{D}$ to $S$ is involutive and $S_{g}, g \in V_{e}$, is a maximal integral manifold of $D^{S}$ and vice versa. We set $\left(\Delta^{-1}\right)_{*} D^{S}=D$. Then $D$ is an involutive distribution on $U_{x_{0}} \times V_{e}$. For each $g \in V_{e}, g$ transforms any maximal integral manifold $S_{h}$ of $D^{S}$ to another maximal integral manifold $S_{g h}$ if $g h \in V_{e}$, which implies that, for any cross-section $\mathrm{X}$ of $D^{S}$ and any $Y \in \mathrm{g}_{s}^{*}$, we have also a cross-section $[\mathrm{X}, Y]$ of $D^{S}$ where $g_{s}^{*}$ is the Lie algebra induced from the action of $G$ on $S$. Therefore by the property $[\beta]$ of $\Delta$, we can also see that, for any cross-section $\mathrm{X}$ of $D$ and any $Y \in \mathrm{g}^{*}$, we have a cross-section $[\mathrm{X}, Y]$ of $D$ where $\mathrm{g}^{*}$ is a Lie algebra induced from the action of $G$ on $N \times G$ as a principal fiber bundle $(N \times G)(N, G, \pi)$. We set $q_{0}=\Delta^{-1}\left(p_{0}\right)$. Then $D$ is a Lie-Vessiot system at $q_{0}$ on $(N \times G)(N, G, \pi)$. Any first integral $\phi$ of $D$ at $q_{0}$ induces a first integral $\varphi=\psi \circ \Delta^{-1}$ of $D^{S}$ at $p_{0}$. Since $\varphi$ is constant on $S_{\omega}$ for any solution $\omega: U_{x_{0}}^{\prime} \rightarrow Q$ of $(A)_{l}$, this completes the proof of Theorem 2.1.

Definition 2.5. Let $\rho^{l}$ be the projection of $J^{l}(N, Q)$ onto $N \times Q$. For a submanifold $S$ of $J^{l}(N, Q)$ we set $\rho_{S}^{l}=\rho^{l} \mid S$, the restriction of $\rho^{l}$ to $S$. A point $p \in S$ is said to be of maximal rank in $S$ if $\left(d \rho_{S}^{l}\right)_{p}$ is of maximal rank. $S$ is said to be of maximal rank if each point of $S$ is of maximal rank.

Corollary 2.1. Let $(A)_{l}$ be a system at $p_{0}$ satisfying $\left[\alpha_{i}\right](i=1,2$, $3)$ and $[\beta]$. We assume that $S$ is of maximal rank. Then we can induce from $(A)_{l}$ a Lie-Vessiot system $D$ at $q_{0}=\Delta^{-1}\left(p_{0}\right)$ on the trivial principal fiber bundle $(N \times G)(N, G, \pi)$ such that we can integrate $(A)_{l}$ at $p_{0}$ by seeking for an arbitrary fundamental system of solutions of $D$ at $q_{0}$.

Proof. Let $\varphi_{1}, \ldots, \varphi_{r}$ be any fundamental system of solutions of $D^{S}$ at $p_{0}$. Then we have the functional determinant $D\left(\varphi_{1}, \ldots, \varphi_{r}\right) / D\left(z_{1}, \ldots\right.$, $\left.z_{q}, w_{1}, \ldots, w_{r-q}\right) \neq 0$ on a neighbourhood $U_{p_{0}}^{S}$ of $p_{0}$ where $\left\{x_{1}, \ldots, x_{n}, z_{1}\right.$, $\left.\ldots, z_{q}, w_{1}, \ldots, w_{r-q}\right\}$ is the coordinate system on $U_{p_{0}}^{S}$ given in the proof of Proposition 2.1. We set $x_{i}^{0}=x_{i}\left(p_{0}\right)(1 \leqq i \leqq n), z_{j}^{0}=z_{j}\left(p_{0}\right)(1 \leqq j \leqq q)$, $w_{k}^{0}=w_{k}\left(p_{0}\right)(1 \leqq k \leqq r-q)$ and $\varphi_{j}\left(x_{1}^{0}, \ldots, x_{n}^{0}, z_{1}^{0}, \ldots, z_{q}^{0}, w_{1}^{0}, \ldots, w_{r-q}^{0}\right)=c_{j}$ 
$(1 \leqq j \leqq r)$. Then by the implicit function theorem we have locally a unique system of functions $f_{z_{j}}(1 \leqq j \leqq q), f_{w_{l k}}(1 \leqq k \leqq r-q)$ of $x_{1}, \ldots, x_{n}$ such that $\varphi_{j}\left(x_{1}, \ldots, x_{n}, f_{z_{1}}(x), \ldots, f_{z_{q}}(x), f_{w_{1}}(x), \ldots, f_{w_{r-q}}(x)\right)=c_{j}(1 \leqq$ $j \leqq r)$ and $f_{z_{j}}\left(x^{0}\right)=z_{j}^{0}(1 \leqq j \leqq q), f_{w_{k}}\left(x^{0}\right)=w_{k}^{0}(1 \leqq k \leqq r-q)$. On the other hand, since $p_{0} \in S$, we have a solution $s_{g}, g \in V_{e}$, of $(A)_{l}$ such that $p_{0}=j_{x_{0}}^{l}\left(s_{g}\right)$. Since $z_{j}\left(s_{g}\left(x^{0}\right)\right)=z_{j}^{0}(1 \leqq j \leqq q), w_{k}\left(s_{g}\left(x^{0}\right)\right)=w_{k}^{0}(1 \leqq k \leqq r-q)$ and $\varphi_{j}\left(x_{1}, \ldots, x_{n}, z_{1}\left(s_{g}(x)\right), \ldots, z_{q}\left(s_{g}(x)\right), w_{1}\left(s_{g}(x)\right), \ldots, w_{r-q}\left(s_{g}(x)\right)\right)=c_{j}(1 \leqq$ $j \leqq r)$, we have $f_{z_{j}}=s_{g}^{j}\left(=z_{j}\left(s_{g}\right)\right)$. Thus from any fundamental system of solutions of $D^{S}$ at $p_{0}$, we can obtain a solution of $(A)_{l}$, that is, we can integrate $(A)_{l}$ at $p_{0}$.

Corollary 2.2. Let $(A)_{l}$ be a system at $p_{0}$ satisfying $\left[\alpha_{i}\right](i=1,2,3)$ and $[\beta]$. We set $S^{0}=\rho^{l}(S) \subset N \times Q$ and assume that $S^{0}$ is a submanifold of $N \times Q$ defined by $z_{i}=\varphi_{1}\left(x_{i}, \cdots, x_{m}, z_{1}, \cdots, z_{t}\right)(t+1 \leqq i \leqq q)$. Then we can induce from $(A)_{l}$ a Lie-Vessiot system $D$ at $q_{0}=\Delta^{-1}\left(p_{0}\right)$ on the trivial principal fiber bundle $(N \times G)(N, G, \pi)$ such that we can integrate $(A)_{l}$ at $p_{0}$ by seeking for an arbitrary fundamental system of solutions of $D$ at $q_{0}$.

Proof. By the same argument, we can obtain $f_{z_{j}}\left(x_{1}, \ldots, x_{n}\right)(1 \leqq$ $j \leqq t$ ) from a fundamental system of solutions of $D$ at $q_{0}$. For $t+1 \leqq$ $i \leqq q$ we set $f_{z_{i}}=\varphi_{i}\left(x_{1}, \ldots, x_{n}, f_{z_{1}}, \ldots, f_{z_{t}}\right)$. Therefore we can obtain a solution of $(A)_{l}$ from a fundamental system of solutions of $D$ at $q_{0}$.

Let $(A)_{l}$ be a $G$-automorphic system satisfying $\left[\alpha_{i}\right](i=1,2,3)$. Let $y_{1}, \ldots, y_{r}$ be linearly independent functions on an open subset $U$ of $S$ where $\operatorname{dim} G=r$. Let $\left\{a_{h}\right\}_{h=1}^{r-1}$ be any family of real numbers. As before we set $y=\left(y_{1}, \ldots, y_{r}\right), U_{y}^{j}=\left\{p \in U_{y}^{j-1} \mid y_{j}(p)=a_{j}\right\}$ and $y_{j}^{j-1}=y_{j} \mid U_{y}^{j-1}$ where $y_{j}^{0}=y_{j}, U_{j}^{0}=U$. We set $g \circ y_{j}^{j-1}(p)=y_{j}^{j-1}(g \circ p)$ and $V_{y}^{j}=\{g \in$ $\left.V_{y}^{j-1} \mid g \cdot y_{j}^{j-1}=y_{j}^{j-1}\right\}, V_{y}^{0}=V_{e}$. Clearly each $g \in V_{y}^{j}$ operates on $U_{y}^{j}$ if $g$ operates on $U$.

Definition 2.6. Let $f: S \rightarrow T$ be a map of a manifold $S$ to a manifold $T$. We set $\operatorname{graph}(f)=\{(p, f(p)) \mid p \in S\} \subset S \times T . \quad \operatorname{graph}(f)$ is called the graph of $f$.

Definition 2.7. Let $D$ be an $m(=\operatorname{dim} S)$ dimensional distribution on 
$S \times \boldsymbol{R}$ defined on a neighbourhood of $(p, t) \in S \times \boldsymbol{R}$. If there exists a local coordinate system $\left\{\alpha_{1}, \ldots, \alpha_{m}, x\right\}$, where $\left\{\alpha_{1}, \ldots, \alpha_{m}\right\}$ (resp. $\{x\}$ ) is a local coordinate system of $S$ at $p$ (resp. of $\boldsymbol{R}$ at $t$ ), such that a local basis $\left\{\mathrm{X}_{1}, \ldots, X_{m}\right\}$ of $D$ at $(p, t)$ is expressed as $X_{j}=\frac{\partial}{\partial \alpha_{j}}+\phi_{j}\left(\alpha_{1}, \ldots, \alpha_{m}\right) \frac{\partial}{\partial x}$, then $D$ is said to be of quadrature type at $(p, t)$.

Definition 2.8. A system $(A)_{l}$ of partial differential equations at $p_{0} \in J^{l}(N, Q)$ satisfying $\left[\alpha_{i}\right](i=1,2,3)$ and $[\beta]$ is said to be solvable if there exists a family of linearly independent functions $\left\{y_{j}\right\}_{j=1}^{r}, r=\operatorname{dim} G$, on a neighbourhood $U$ of $p_{0}$ in $S$ which satisfies, for a family of real numbers $\left\{a_{h}\right\}_{h=1}^{r-1}$, the following conditions;

[1] We can induce from $(A)_{l}$ an $m_{j}\left(=\operatorname{dim} U_{y}^{j}\right)$ dimensional distribution $D^{j}$ on $U_{y}^{j} \times \boldsymbol{R}$ such that $D^{j}$ is of quadrature type at any point in $U_{y}^{j} \times \boldsymbol{R}$ and for any $g \in V_{y}^{j}$ the graph of $g \cdot y_{j+1}^{j}$ is an integral manifold of $D^{j}(j=0, \ldots, r-1)$.

[2] There exists a solution $\omega$ of $(A)_{l}$ defined on $U_{x_{0}}^{\omega} \subset U_{x_{0}}$ such that, if we set $S_{\omega}=\left\{j_{x}^{l}(\omega) \mid x \in U_{x_{0}}^{\omega}\right\}$, the function $y_{j} \mid S_{\omega}$, the restriction of $y_{j}$ to $S_{\omega}$, is constant for each $j(j=1, \ldots, r-1)$ and $y_{j} \mid S_{\omega}=a_{j}$.

We shall call $\left\{y_{j}\right\}_{j=1}^{r}$ satisfying $[1],[2]$, a fundamental family of functions of $(A)_{l}$.

Theorem 2.2. Let $(A)_{l}$ be a system of partial differential equations at $p_{0} \in J^{l}(N, Q)$ satisfying $\left[\alpha_{i}\right](i=1,2,3)$ and $[\beta]$. Then the following two statements are equivalent;

[i] $G$ is solvable. [ii] $(A)_{l}$ is solvable.

Proof. First of all, we shall prove $[\mathrm{i}] \Rightarrow[\mathrm{ii}]$. By Theorem 2.1, we can find a Lie-Vessiot system $D$ at $q_{0}=\Delta^{-1}\left(q_{0}\right)$ on $(N \times G)(N, G, \pi)$. Since we have a basis $\left\{X_{h}\right\}_{h=1}^{m}$ of $D$ at $p_{0}$ such that $\left[\mathrm{g}^{*}, X_{h}\right]=0(1 \leqq$ $h \leqq m$ ), for any ideal $\mathfrak{h}$ of $\mathfrak{g}, E=[\mathfrak{h} * \cup D]$ (the distribution on $U_{x_{0}} \times V_{e}$ generated by $\mathfrak{h}^{*}$ and $D$ ) is involutive. Since $\mathfrak{g}$ is solvable, we have a sequence of subalgebras $\mathrm{g}=\mathrm{g}_{0} \supset \mathrm{g}_{1} \supset \cdots \supset \mathrm{g}_{r-1} \supset \mathrm{g}_{r}=\{0\}$ such that $\operatorname{dim} \mathrm{g}_{j}-$ $\operatorname{dim} \mathfrak{g}_{j+1}=1$ and $\mathfrak{g}_{j+1}$ is an ideal of $\mathfrak{g}_{j}(0 \leqq j \leqq r-1)$. We set $E_{j}=\left[\mathfrak{g}_{j}^{*} \cup\right.$ 
$D](0 \leqq j \leqq r)$. Let $s_{1}, \cdots, \diamond_{r}$ be any fundamental system of solutions of $D=E_{r}$. We set $y_{j}={ }_{s_{j}} \circ \Delta^{-1}(1 \leqq j \leqq r)$. Then by Theorem 2.1 for any solution $\omega$ of $(A)_{l}, y_{j} \mid S_{\omega}$ is constant for each $1 \leqq j \leqq r$. Therefore, if we set $y_{j} \mid S_{\omega}=a_{j}(1 \leqq j \leqq r-1),\left\{y_{j}\right\}_{j=1}^{r}$ satisfies [2]. We shall prove that we can find a fundamental system of solutions $\left\{s_{j}\right\}_{j=1}^{r}$ of $D$ such that [1] is also satisfied. We choose a basis $V_{1}, \ldots, V_{r}$ such that $\left\{V_{k}\right\}_{k=j}^{r}$ is a basis of $\mathrm{g}_{j-1}^{*}$. By Theorem 1.1, there exists a function $\triangleleft_{1}$ on $U_{x_{0}} \times V_{e}$ such that $x_{1} \cdot s_{1}=\cdots=X_{m} \cdot s_{1}=V_{1} \cdot s_{1} \cdots=V_{r-1} \cdot s_{1}=0$ and $V_{r} \cdot s_{1}=1$. By using a local coordinate system $\left\{\alpha_{1}, \ldots, \alpha_{n+r}\right\}$ on $U_{x_{0}} \times V_{e}$, the system

$$
\left\{\begin{array}{l}
X_{h \cdot\lrcorner_{1}}=V_{k \cdot\lrcorner_{1}}=0 \quad(1 \leqq h \leqq m, 2 \leqq k \leqq r) \\
V_{1 \cdot\lrcorner_{1}}=1
\end{array}\right.
$$

is expressed as

$$
\left\{\begin{array}{l}
\sum_{j=1}^{n+r} \xi_{k}^{j} \frac{\partial \jmath_{1}}{\partial \alpha_{j}}=0 \quad(1 \leqq k \leqq n+r-1) \\
\sum_{j=1}^{n+r} \xi_{n+r}^{j} \frac{\partial \diamond_{1}}{\partial \alpha_{j}}=1
\end{array}\right.
$$

We have therefore $\frac{\partial_{\unlhd}}{\partial \alpha_{j}}=\phi_{j}\left(\alpha_{1}, \ldots, \alpha_{n+r}\right)(1 \leqq j \leqq n+r)$. Let $\left\{\alpha_{1}, \ldots\right.$, $\left.\alpha_{n+r}, x\right\}$ be accordinate system on $U_{x_{0}} \times V_{e} \times \boldsymbol{R}$ and we denote by $D^{0}$ the $(n+r)$-dimensional distribution on $S \times \boldsymbol{R}$ generated by $\tilde{\Delta}_{*}\left(\frac{\partial}{\partial \alpha_{j}}+\phi_{j} \frac{\partial}{\partial x}\right)$ $(1 \leqq j \leqq n+r)$ where $\tilde{\Delta}$ is the diffeomorphism of $U_{x_{0}} \times V_{e} \times \boldsymbol{R}$ onto $S \times \boldsymbol{R}$ defined by $\tilde{\Delta}(x, g, t)=(\Delta(x, g), t)$. Clearly the graph of $s_{1} \circ \Delta^{-1}$ is a maximal integral manifold of $D^{0}$.

We shall prove that, for any $g \in V_{e}$, the graph of $g \cdot\left(\alpha_{1} \circ \Delta^{-1}\right)$ is also an integral manifold of $D^{0}$. Since $s_{1}$ is a first integral of the involutive distribution $E_{1}$, and since we have $\left[g^{*}, E_{1}\right] \subset E_{1}, g \cdot s_{1}$ is also a first integral of $E_{1}, g \in V_{e}$. On the other hand $\operatorname{codim} E_{1}=1$. Therefore we have a function $H_{g}$ of one variable such that $g \cdot s_{1}=H_{g}\left(s_{1}\right)$. Since we have $\operatorname{dim} \mathrm{g}^{*} / \mathrm{g}_{1}^{*}=1$, the local transformation group on $\boldsymbol{R}$ consisting of $H_{g}$, $g \in V_{e}$, is commutative. Hence we have $H_{e_{t}}\left(H_{g}\left(\jmath_{1}\right)\right)=H_{g}\left(H_{e_{t}}\left(\jmath_{1}\right)\right)$ where $e_{t}=\exp t V_{1}$. We have then $V_{1}\left(g \cdot \diamond_{1}\right)=g\left(V_{1} \bullet_{\jmath_{1}}\right)$. Therefore we have also 


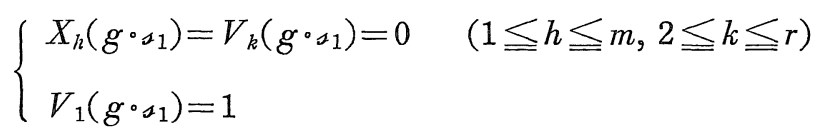

and therefore we have

$$
\frac{\partial\left(g \cdot \jmath_{1}\right)}{\partial \alpha_{j}}=\phi_{j}\left(\alpha_{1}, \ldots, \alpha_{n+r}\right) \quad(1 \leqq j \leqq n+r)
$$

This implies that the graph of $g\left(\sigma_{1} \circ \Delta^{-1}\right)$ is also an integral manifold of $D^{0}$.

Let $s_{1}, \ldots, s_{r}$ be a fundamental system of solutions of $D$ such that we have

$$
\left\{\begin{array}{l}
X_{h^{\circ} \diamond_{j+1}^{j}}^{j}=V_{k^{\circ} \diamond_{j+1}^{j}}^{j}=0 \quad(1 \leqq h \leqq m, j+2 \leqq k \leqq r) \\
V_{j+1}^{j} \bullet^{j} \otimes_{j+1}^{j}=1
\end{array}\right.
$$

on $\left(U_{x_{0}} \times V_{e}\right)^{j} \quad(0 \leqq j \leqq r-1)$. We choose a coordinate system $\left\{\alpha_{1}^{j}, \cdots\right.$, $\left.\alpha_{n+r-j}^{j}\right\}$ on $S_{j}^{j}$. Then we have from $(*)_{j}$

$$
\frac{\partial_{s_{j+1}^{j}}^{j}}{\partial \alpha_{k}^{j}}=\phi_{k}^{j}\left(\alpha_{1}^{j}, \ldots, \alpha_{n+r-j}^{j}\right) \quad(1 \leqq k \leqq n+r-j)
$$

for each $0 \leqq j \leqq r-1$. Therefore by similar method we get an $(n+r-j)$ dimensional distribution $D^{j}$ on $S S_{2}^{j} \times \mathbb{R}$ of quadrature type such that, if we set $V_{e}^{j}=V_{e} \cap \exp g_{j}$, the graph of $g \cdot \cdot_{j+1}^{j}$ is an integral manifold of $D_{j}$ for any $g \in V_{e}^{j}$. Note that we have $V_{e}^{j}=V_{2}^{j}$. This implies $\left\{a_{j} \circ \Delta^{-1}\right\}_{j=1}^{r}$ is a fundamental family of functions of $(A)_{l}$. This completes the proof of $[\mathrm{i}] \Rightarrow[\mathrm{ii}]$.

Conversely we shall prove $[$ ii $] \Rightarrow[\mathrm{i}]$. We denote also by $\mathrm{g}^{*}$ the Lie algebra induced from the Lie algebra $\mathrm{g}$ of $G$ by the action of $G$ on $J^{l}(N$, $Q)$. We set $\mathrm{g}_{(0)}^{*}=\mathrm{g}^{*}$ and inductively we set $\mathrm{g}_{(j)}^{*}=\left\{X \in \mathrm{g}_{(j-1)}^{*} \mid X \cdot y_{j}^{j-1}=\right.$ $0\} \mid U_{y}^{j}(j=0, \ldots, r-1)$ where $\left\{y_{j}\right\}_{j=1}^{r}$ is a fundamental family of functions of $(A)_{l}$. Note that, since $V_{e}$ acts freely on $S$, the restriction $\operatorname{map} \mid U_{y}^{j}:\left\{X \in g_{(j-1)}^{*} \mid X \cdot y_{j}^{j-1}=0\right\} \rightarrow g_{(j)}^{*}$ is an isomorphism. By pulling back $\mathrm{g}_{(j)}^{*}$ to a Lie subalgebra $\mathrm{g}_{j}^{*}$ of $\mathrm{g}^{*}$ through these restriction maps, we get a sequence of Lie subalgebras $g^{*} \supset g_{1}^{*} \supset \cdots \supset g_{k}^{*} \supset \cdots$ such that $g_{k}^{*}$ is 
isomorphic to $\mathrm{g}_{(k)}^{*}$. Therefore we get also a chain of Lie subalgebras $\mathrm{g} \supset \mathrm{g}_{1} \supset \cdots \supset \mathrm{g}_{k} \supset \cdots$ such that $\mathrm{g}_{k}$ is isomorphic to $\mathrm{g}_{k}^{*}$. We have $V_{y}^{j}=V_{e} \cap$ $\exp _{j}(j=1,2, \ldots)$. First of all we prove that $g \cdot y_{j+1}^{j}=y_{j+1}^{j}+c_{g}^{j}, g \in$ $V_{y}^{j}$, where $c_{g}^{j}$ is constant. By the assumption $[1]$, the graph of $g \cdot y_{j+1}^{j}$ is an integral manifold of $D^{j}$. Since $D^{j}$ is of quadrature type, there exists a local basis $\left\{X_{1}^{j}, \ldots, X_{m_{j}}^{j}\right\}$ such that, for a coordinate system $\left\{\alpha_{1}^{j}\right.$, $\left.\cdots, \alpha_{m_{j}}^{j}\right\}$ on $U_{y}^{j}, X_{k}^{j}$ is expressed as $X_{k}^{j}=\frac{\partial}{\partial \alpha_{k}^{j}}+\phi_{k}^{j}\left(\alpha_{1}^{j}, \ldots, \alpha_{m_{j}}^{j}\right) \frac{\partial}{\partial x}(1 \leqq k$ $\left.\leqq m_{j}\right)$. Therefore $g \cdot y_{j+1}^{j}, g \in V_{y}^{j}$, satisfies $\frac{\partial\left(g \cdot y_{j+1}^{j}\right)}{\partial \alpha_{k}^{j}}=\phi_{k}^{j} \quad\left(\alpha_{1}^{j}, \ldots, \alpha_{m_{j}}^{j}\right)$ for each $k$. Fix an integer $k, 1 \leqq k \leqq m_{j}$. Considering $\alpha_{h}^{j}(h \neq k)$ as parameters of an ordinary differential equation $\frac{d\left(g \cdot y_{j+1}^{j}\right)}{d \alpha_{k}^{j}}=\phi_{k}^{j} \quad\left(\alpha_{1}^{j}, \ldots, \alpha_{m_{j}}^{j}\right)$, we get $g \cdot y_{j+1}^{j}=y_{j+1}^{j}+c_{g}^{j}\left(\ldots, \alpha_{h}^{j}, \cdots\right), h \neq k$. Since $k$ runs over the set of integers $\left\{1,2, \ldots, m_{j}\right\}, c_{g}^{j}$ must be constant. We shall next show that $\mathrm{g}_{j+1}^{*}$ is an ideal of $\mathrm{g}_{j}^{*}$. Let $\sigma$ (resp. $g$ ) be any element of $V_{y}^{j}$ (resp. $V_{y}^{j+1}$ ). Then we have $\left(\sigma^{-1} \cdot g \cdot \sigma\right) \cdot y_{j+1}^{j}=(\sigma)^{-1} \cdot g \cdot \sigma \cdot y_{j+1}^{j}=(\sigma)^{-1} \cdot g\left(y_{j+1}^{j}+c_{\sigma}\right)=$ $(\sigma)^{-1}\left(y_{j+1}^{j}+c_{\sigma}\right)=y_{j+1}^{j}$. This implies that $\mathrm{g}_{j+1}^{*}$ is an ideal of $\mathrm{g}_{j}^{*}$. By [2] we have a solution $\omega$ of $(A)_{l}$ such that $y_{j} \mid S_{\omega}=a_{j}(1 \leqq j \leqq r-1)$. Therefore we have $S_{\omega} \cap U \subset U_{y}^{j}(0 \leqq j \leqq r-1)$. Since any $g \in V_{y}^{j}$ leaves $y_{1}^{j}, \ldots, y_{j}^{j}$ invariant, that is $g \cdot y_{k}^{j}=y_{k}^{j}(1 \leqq k \leqq j)$, we have $S_{\omega}^{g} \cap U \subset U_{y}^{j}$, $g \in V_{y}^{j}$, where $S_{\omega}^{g}=g \cdot S_{\omega}$. Therefore we get $U_{y}^{j} \supset \bigcup_{g \in V_{y}^{j}} S_{\omega}^{g} \cap U$. On the other hand if $g \in V_{y}^{j-1}, \notin V_{y}^{j}$, then we have $g \cdot y_{j}^{j-1} \neq y_{j}^{j-1}$ and therefore $c_{g}^{j-1} \neq 0$. This implies that $y_{j}^{j-1}\left|S_{\omega}^{g} \cap U \neq y_{j}^{j-1}\right| S_{\omega} \cap U$. Therefore $S_{\omega}^{g} \cap$ $U \succ U_{y^{j}}^{j}$. We get, therefore, $S_{\omega}^{g} \cap U \subset U_{y}^{j}$ if and only if $g \in V_{y}^{j}$. We shall show that $U_{y}^{j}$ is a union of some $S_{\omega}^{g} \cap U, g \in V_{e}$. Since we have $g \cdot y_{1}=$ $y_{1}+c_{g}^{1}$ for any $g \in V_{e}, y_{1}$ is constant on each $S_{\omega}^{g} \cap U, g \in V_{e}$. On the other hand, since $(A)_{l}$ is $G$-automorphic, we have $U=\bigcup_{g \in V_{e}} S_{\omega}^{g} \cap U$ (disjoint union). This implies that $U_{y}^{1}$ is a (disjoint) union of some $S_{\omega}^{g} \cap U, g \in$ $V_{e}$. Therefore we get $U_{y}^{1}=\bigcup_{g \in V_{y}^{1}} S_{\omega}^{g} \cap U$. Since $g \cdot y_{2}^{1}=y_{2}^{1}+C_{g}^{2}, g \in V_{y}^{1}$, $y_{\frac{1}{2}}^{1}$ is constant on each $S_{\omega}^{g} \cap U C U_{y}^{1}$. This implies $U_{y}^{2}$ is also a (disjoint) union of some $S_{\omega}^{g} \cap U, g \in V_{e}$ which implies $U_{y}^{2}=\bigcup_{g \in V_{y}^{2}} S_{\omega}^{g} \cap U$. Similarly $U_{y}^{j}$ is a union of some $S_{\omega}^{g} \cap U, g \in V_{e}(0 \leqq j \leqq r-1)$. This implies that 
$U_{y}^{j}=\bigcup_{g \in V_{y}^{\prime}} S_{\omega}^{g} \cap U$. Now we have $\operatorname{dim} U_{y}^{j}-\operatorname{dim} U_{y}^{j+1}=1$ and since $(A)_{l}$ is $G$-automorphic, we have $\operatorname{dim} \bigcup_{g \in V_{y}^{j}} S_{\omega}^{g} \cap U=\operatorname{dim} S_{\omega}+\operatorname{dim} g_{j}^{*}$. Therefore we get $\operatorname{dim} \mathrm{g}_{j}^{*}-\operatorname{dim} \mathrm{g}_{j+1}^{*}=1$. This proves that $\mathrm{g}$ is solvable. This complete the proof of Theorem 2.2 .

Corollary 2.3. Let $(A)_{l}$ be a system at $p_{0} \in J^{l}(N, Q)$ satisfying $\left[\alpha_{i}\right]$ $(i=1,2,3)$ and $[\beta]$. Then $(A)_{l}$ is solvable if and only if there exists a family of linearly independent functions $\left\{y_{j}\right\}_{j=1}^{r}, r=\operatorname{dim} G$, on a neighbourhood $U$ of $p_{0}$ in $S$ which satisfies, for a family of real numbers $\left\{a_{h}\right\}_{h=1}^{r-1}$, the following conditions;

[1] We have $g \cdot y_{j+1}^{j}=H_{g}^{j}\left(y_{j+1}^{j}\right), g \in V_{y}^{j}$, for a function $H_{g}^{j}$ of one variable depending on $g$ and $j(j=0, \ldots, r-1)$.

[2] There exists a solution $\omega$ of $(A)_{l}$ such that $y_{j} \mid S_{\omega}=a_{j}(j=1, \ldots$, $r-1)$ and such that if, $g \in V_{y}^{j}, g \cdot y_{j+1}^{j} \neq y_{j+1}^{j}$, then we have $y_{j+1}^{j} \mid S_{\omega} \cap$ $U \neq y_{j+1}^{j} \mid S_{\omega}^{g} \cap U(j=0, \ldots, r-1)$.

Proof. We already showed in the proof of Theorem 2.2. that if $(A)_{l}$ is solvable, then any fundamental family of functions $\left\{y_{j}\right\}_{j=1}^{r}$ of $(A)_{l}$ satisfied [1], [2]. Conversely let $\left\{y_{j}\right\}_{j=1}^{r}$ be a family of linearly independent functions on $U$ satisfying [1] and [2]. We have only to show that $G$ is solvable. First of all we shall prove that $U_{y}^{j}$ is a disjoint union of some $S_{\omega}^{g} \cap U, g \in V_{e}$. Since we have $g \cdot y_{1}=H_{g}^{1}\left(y_{1}\right)$ for any $g \in V_{e}$, $y_{1}$ is constant on each $S_{\omega}^{g} \cap U, g \in V_{e}$. Since $(A)_{l}$ is $G$-automorphic, we have $U=\bigcup_{g \in V_{e}} S_{\omega}^{g} \cap U$ (disjoint union). Therefore $U_{y}^{1}$ is a disjoint union of some $S_{\omega}^{g} \cap U, g \in V_{e}$. We have clearly $U_{y}^{1} \supset \bigcup_{g \in V_{y}^{1}} S_{\omega}^{g} \cap U . \quad$ If $g \in V_{e}$ and $\notin V_{y}^{1}$, then $g \cdot y_{1} \neq y_{1}$. Therefore by the assumption $[2], y_{1} \mid S_{\omega}^{g} \cap$ $U \neq y_{1} \mid S_{\omega} \cap U$. Hence we get $S_{\omega}^{g} \cap U \subset U_{y}^{1}$ if and only if $g \in V_{y}^{1}$. This implies $U_{y}^{1}=\bigcup_{g \in V_{y}^{1}} S_{\omega}^{g} \cap U$. By similar considerations we have $U_{y}^{j}=\bigcup_{g \in V_{y}^{j}}$ $S_{\omega}^{g} \cap U$ for $0 \leqq j \leqq r-1$. We have $\operatorname{dim} U_{y}^{j}-\operatorname{dim} U_{y}^{j+1}=1$ and since $(A)_{l}$ is $G$-automorphic, we have $\operatorname{dim} \bigcup_{g \in V_{y}^{j}} S_{\omega}^{g} \cap U=\operatorname{dim} S_{\omega}+\operatorname{dim} g_{j}^{*}$. Therefore we get $\operatorname{dim} g_{j}^{*}-\operatorname{dim} g_{j+1}^{*}=1$. Let $\sigma$ (resp. $g$ ) be any element of $V_{y}^{j}$ (resp. $\left.V_{y}^{j+1}\right)$. Then $\left(\sigma^{-1} \cdot g \cdot \sigma\right) \cdot y_{j+1}^{j}=(\sigma)^{-1} \cdot g\left(H_{\sigma}^{j+1}\left(y_{j+1}^{j}\right)\right)=(\sigma)^{-1} H_{\sigma}^{j+1}\left(y_{j+1}^{j}\right)$ 
$=y_{j+1}^{j}$. This implies that $\mathrm{g}_{j+1}^{*}$ is an ideal of $\mathrm{g}_{j}^{*}$. Therefore $\mathrm{g}$ is solvable. By Theorem 2.2, $(A)_{l}$ is solvable. This completes the proof of Corollary 2.3.

Corollary 2.4. Let $(A)_{l}$ be a system at $p_{0} \in J^{l}(N, Q)$ satisfying $\left[\alpha_{i}\right]$ $(i=1,2,3)$ and $[\beta]$. Let $D$ be the Lie-Vessiot system at $q_{0}=\Delta^{-1}\left(p_{0}\right)$ on $(N \times G)(N, G, \pi)$ induced from $(A)_{l}$ by Theorem 2.1. If $(A)_{l}$ is solvable, then there exists a fundamental family of functions of $(A)_{l}$ on a neighbourhood $U$ of $p_{0}$ in $S$ which satisfies the following conditions:

[1] There exists a basis $\left\{V_{k}\right\}_{k=1}^{r}$ of $\mathrm{g}^{*}$ such that we have

$$
\left\{\begin{array}{l}
V_{k+1} \cdot\left(y_{k+1} \circ \Delta\right)=1 \\
V_{k+j} \cdot\left(y_{k+1} \circ \Delta\right)=0 \quad(2 \leqq j \leqq r-k)
\end{array}\right.
$$

for $0 \leqq k \leqq r-1$.

[2] $\left\{y_{k} \circ \Delta\right\}_{k=1}^{r}$ is a fundamental system of solutions of $D$ at $q_{0}$.

Proof. By Theorem 2.2, $G$ is solvable. Then the Lie-Vessiot system $D$ at $q_{0}$ on $(N \times G)(N, G, \pi)$ is solvable. By Theorem 1.1, there exist a fundamental system of solutions $\left\{\alpha_{k}\right\}_{k=1}^{r}$ of $D$ at $q_{0}$ and a basis $\left\{V_{k}\right\}_{k=1}^{r}$ of $\mathrm{g}^{*}$ such that we have

$$
\left\{\begin{array}{l}
V_{k+1} \cdot \rtimes_{k+1}=1 \\
V_{k+j} \cdot \rtimes_{k+1}=0 \quad(2 \leqq j \leqq r-k)
\end{array}\right.
$$

for $0 \leqq k \leqq r-1$. We put $y_{j}=\triangleleft_{j} \circ \Delta^{-1}(j=1,2, \ldots, r)$. Since $g^{*}$ is solvable, we have a sequence of subalgebras $g^{*}=g_{0}^{*} \supset g_{1}^{*} \supset \ldots \supset g_{r-1}^{*} \supset g_{r}^{*}=\{0\}$ such that $\operatorname{dim} \mathrm{g}_{j}^{*}-\operatorname{dim} \mathrm{g}_{j+1}^{*}=1$ and $\mathrm{g}_{j+1}^{*}$ is an ideal of $\mathrm{g}_{j}^{*}$. Note that, in Theorem 1.1, we chose a basis $\left\{V_{k}\right\}_{k=1}^{r}$ of $\mathrm{g}^{*}$ such that $\left\{V_{k}\right\}_{k=j}^{r}$ is a basis of $\mathrm{g}_{j-1}^{*}$, from which, as is proved in Theorem 2.2, it follows that $\left\{y_{j}\right\}_{j=1}^{r}$ is a fundamental family of functions of $(A)_{l}$.

Corollary 2.5. Let $(A)_{l}$ be a system satisfying $\left[\alpha_{i}\right](i=1,2,3)$ and $[\beta]$. Moreover we assume that $S$ is of maximal rank. If $(A)_{l}$ is solvable, then we can induce a fundamental family of functions $\left\{y_{j}\right\}_{j=1}^{r}$ of $(A)_{l}$ by quadratures such that we can obtain a general solution of $(A)_{l}$ by applying to $\left\{y_{j}\right\}_{j=1}^{r}$ the implicit function theorem. 
Proof. By Corollary 2.4, there exists a fundamental famliy of functions $\left\{y_{j}\right\}_{j=1}^{r}$ such that $\left\{y_{j} \circ \Delta\right\}_{j=1}^{r}$ satisfies $[1]$ and [2] in Corollary 2.4, which implies, by Corollary 1.1, that $\left\{y_{j} \circ \Delta\right\}_{j=1}^{r}$ is obtained by quadrature. By Corollary 2.1, we can obtain a general solution of $(A)_{l}$ by applying to $\left\{y_{j}\right\}_{j=1}^{r}$ the implicit function theorem.

Corollary 2.6. Let $(A)_{l}$ be a system satisfying $\left[\alpha_{i}\right](i=1,2,3)$ and

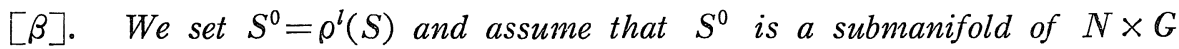
defined by $z_{i}=\varphi_{i}\left(x_{1}, \cdots, x_{n}, z, \cdots, z_{t}\right)(t+1 \leqq i \leqq q)$. If $(A)_{l}$ is solvable, then we can induce a fundamental family of functions $\left\{y_{j}\right\}_{j=1}^{r}$ of $(A)_{l}$ by quadratures such that we can obtain a general solution of $(A)_{l}$ by applying to $\left\{y_{j}\right\}_{j=1}^{r}$ the implicit function theorem.

Proof. Using Corollary 2.2 in place of Corollary 2.1, Corollary 2.6 follows immediately.

\section{§3. Examples}

Example 1. We shall consider Riccati's differential equation $\frac{d z}{d x}=$ $\eta_{1}(x) z^{2}+\eta_{2}(x) z+\eta_{3}(x) . \quad$ We set $\quad X_{1}=z^{2} \frac{d}{d z}, \quad X_{2}=z \frac{d}{d z}$ and $X_{3}=\frac{d}{d z}$. Then $\mathrm{g}=\left\{\sum_{i=1}^{3} c_{i} X_{i} \mid c_{i} \in \boldsymbol{R}\right\}$ is a Lie algebra. Note that $\mathrm{g}$ is the Lie algebra of the projective transformatiton group on the 1-dimensional projective space and therefore simple. We set $X_{*}=\frac{\partial}{\partial x}+\eta_{1} \cdot X_{1}+\eta_{2} \cdot X_{2}+\eta_{3} \cdot X_{3}$ and denote by $G$ a connected Lie group with the Lie algebra g. Then, by considering $X_{i}(i=1,2,3)$ as a right invariant vector field on $G, X_{*}$ is a vector field on $\mathbb{R} \times G$. We can make $G$ a Lie transformation group on $G$ by $g * \alpha=\alpha \cdot g^{-1}(\operatorname{resp} g \circ \alpha=g \circ \alpha) \alpha \in G, g \in G$ which we denote by $G^{*}$ (resp. $\left.G_{*}\right)$. Let $\pi$ be the projection of $\mathbb{R} \times G$ onto $\mathbb{R}$. Then $(\mathbb{R} \times G)(\mathbb{R}$, $\left.G^{*}, \pi\right)$ (resp. $\left.(\boldsymbol{R} \times G)\left(\mathbb{R}, G_{*}, \pi\right)\right)$ is a principal fiber bundle. We denote by $\mathrm{g}^{*}$ (resp. $\mathrm{g}_{*}$ ) the Lie algebra induced from the action of $G^{*}$ (resp. $G_{*}$ ) on $\mathbb{R} \times G$. Then any element of $G^{*}$ commutes with all elements of $G_{*}$ as a transformation on $\mathbb{R} \times G$. Therefore we get $\left[\mathrm{g}^{*}, \mathfrak{g}_{*}\right]=0$. This im- 
plies that we have $\left[X_{*}, \mathrm{~g}^{*}\right]=0$, for any right invariant vector field on $G$ is naturally considered as an element of $\mathrm{g}^{*}$.

We denote by $D$ the distribution on $\mathbb{R} \times G$ generated by $X_{*}$. Then $D$ is a Lie-Vessiot system at any point $p$ on $(\mathbb{R} \times G)\left(\mathbb{R}, G^{*}, \pi\right)$.

Let $p_{0}=\left(t_{0}, g_{0}\right)$ be any point $\in \mathbb{R} \times G$ and let

$$
\begin{aligned}
\frac{d z_{1}}{d x} & =F_{1}\left(x, z_{1}, z_{2}, z_{3}\right) \\
\mathfrak{N}(A)_{1}: \frac{d z_{2}}{d x} & =F_{2}\left(x, z_{1}, z_{2}, z_{3}\right) \\
\frac{d z_{3}}{d x} & =F_{3}\left(x, z_{1}, z_{2}, z_{3}\right)
\end{aligned}
$$

be the system of ordinary differential equations at $\tilde{p}_{0} \in J^{1}(\mathbb{R}, G), \tilde{p}_{0}=$ $\rho_{0}^{1}\left(p_{0}\right)$, such that we have $\left(d z_{i}-F_{i} \bullet d x\right)\left(X_{*}\right)=0$ at any point of a neighbourhood of $p_{0}$, where $\{x\}$ (resp. $\left\{z_{1}, z_{2}, z_{3}\right\}$ ) is a local coordinate system at $t_{0}$ (resp. $\left.g_{0}\right)$. We shall show that $\mathfrak{N}(A)_{1}$ satisfies $\left[\alpha_{i}\right](i=1,2$, $3)$; Let $\left(z_{1}^{0}, z_{2}^{0}, z_{3}^{0}\right)$ be a solution of $\mathfrak{N}(A)_{1}$ defined on $U_{t_{0}}$. We set $s=$ $\left(z_{1}^{0}, z_{2}^{0}, z_{3}^{0}\right)$. Then $s$ is a map of $U_{t_{0}} \rightarrow G$. We set $\bar{s}(x, g)=g * s(x)$. Since $D$ is a Lie-Vessiot system at $p_{0}$ on $(\mathbb{R} \times G)\left(\mathbb{R}, G^{*}, \pi\right)$, both $s\left(U_{t_{0}}\right)$ and $g * s\left(U_{t_{0}}\right)$ are integral manifolds of $D$, that is, $g * s$ is also a solution of $\mathfrak{R}(A)_{1}$ if $g$ is in a neighbourhood $V_{e}$ of the unit of $G$. Since any integral manifold of $D$ contained in a neighbourhood of $p_{0}$ is uniquely expressed as $g * s\left(U_{t_{0}}\right), g \in V_{e}, \mathfrak{N}(A)_{1}$ is $G^{*}$-automorphic. Since $1 \times s$ is a local cross-section of $(\mathbb{R} \times G)\left(\mathbb{R}, G^{*}, \pi\right)$ where 1 is the identity of $\mathbb{R}, j^{1}(\bar{s})$ : $U_{t_{0}} \times V_{e} \rightarrow J^{1}(\mathbb{R}, G)$ is an embedding. Moreover $S=j^{1}(\bar{s})\left(U_{t_{0}} \times V_{e}\right)$ is of maximal rank.

Example 2. Let $G$ be a Lie transformation group acting effectively on a manifold $M$. Then $G$ acts on the space of $l$-jets $J^{l}(M, M)$ natural1y. Then, for a sufficiently large $l, G$ acts freely on $J^{l}(M, M)$, or more precisely, there exists a neighbourhood $V_{e}$ of the unit element of $G$ such that each element of $V_{e}$ acts freely on $J^{l}(M, M)$. We choose and fix an element $g_{0} \in G$. We set $\bar{s}(x, g)=g \cdot g_{0}(x)$. Then $\bar{s}$ is a map of $M \times G$ to $M$. Since $V_{e}$ acts freely on $J^{l}(M, M), j^{l}(\bar{s})$ is an embedding of $M \times$ 
$V_{e}$ into $J^{l}(M, M)$. If $G$ acts transitively on $M, S=j^{l}(\bar{s})\left(M \times V_{e}\right)$ is of maximal rank. Let $(A)_{l}$ be the system of defining equations at $p_{0} \in$ $J^{l}(M, M)$ of the Lie transformation group $G$ acting on $J^{l}(M, M)$. Then $(A)_{l}$ is $G$-automorphic.

Example 3. Let $\left(P_{i}, M_{i},\{e\}\right)$ be an $\{e\}$-structure on $M_{i}$ and $\omega_{i}$ be the basic form of $\left(P_{i}, M_{i},\{e\}\right)(i=1,2)$. We assume that $\left(P_{1}, M_{1},\{e\}\right)$ is locally isomorphic to $\left(P_{2}, M_{2},\{e\}\right)$ at any point $\left(x_{1}, x_{2}\right) \in M_{1} \times M_{2}$. We denote by $\Gamma$ (resp. $\tilde{\Gamma})$ the set of all local isomorphisms of $\left(P_{1}, M_{1}\right.$, $\{e\})$ to $\left(P_{2}, M_{2},\{e\}\right)$ (resp. the set of all local diffeomorphisms $\psi$ of $P_{1}$ to $P_{2}$ with $\left.\psi^{*} \omega_{2}=\omega_{1}\right)$. Then it is well-known that the natural lifting of an element of $\Gamma$ gives a $1-1$ correspondence between $\Gamma$ and $\tilde{\Gamma}$ (cf. Singer, I. M. and S. Sternberg, The infinite groups of Lie and Cartan, J. Analyse Math. 15 (1965), 1-114).

Let $G$ be the automorphism group of $\left(P_{2}, M_{2},\{e\}\right)$ and assume that any local automorphism of $\left(P_{2}, M_{2},\{e\}\right)$ is a restriction of an element of $G$. We denote by $(A)_{1}$ the system of partial differential equations at $p_{0} \in J^{1}\left(P_{1}, P_{2}\right)$ given by $\phi^{*} \omega_{2}=\omega_{1}$. Let $\phi$ be any local diffeomorphism of a neighbourhood $U_{p_{1}}$ of $p_{1} \in P_{1}$ to a neighbourhood $U_{p_{2}}$ of $p_{2} \in P_{2}$ with $\phi^{*} \omega_{2}=\omega_{1}$ and $\phi\left(p_{1}\right)=p_{2}$. We set $\bar{s}(p, g)=g \cdot \phi(p),(p, g) \in U_{p_{1}} \times V_{e}$. Then $(A)_{1}$ is $G$-automorphic and $j^{1}(\bar{s})$ is an embedding of $U_{p_{1}} \times V_{e}$ into $J^{1}\left(P_{1}, P_{2}\right)$. Moreover if $G$ acts transitively on $P_{2}, S=j^{1}(\bar{s})\left(U_{p_{1}} \times V_{e}\right)$ is of maximal rank.

\section{References}

[1] Cartan, E., Sur la réduction à sa forme canonique de la structure d'un groupe de transformation fini et continu, Oeuvres Complètes, Vol. 1, Part 1.

[2] Lie, S., Allgemeine Untersuchungen über Differentialgleichungen, die eine continuirliche endliche Gruppe gestaten, Christiania, 5, Juli 1884.

[3] Matsuda, M., Cartan-Kuranishi's prolongation of differential systems combined with that of Lagrange and Jacobi, Publ. Res. Inst. Math. Sci. Kyoto Univ. Ser. A, 3 (1967), 69-84.

[4] Vessiot, E., D'équations différentielles du première ordre qui ont des fondamentaux d'intégrales, Ann. de Toulouse, 8, 1894. 